Fetal motor activity was assessed by real time ultrasound scanning. All observed movements were punched directly on to computer tape. The beginning and end of each movement was recorded so as to measure the amount of time the fetus was moving in each obervation period. The number of fetal movements was also calculated and expressed as the mean/30-min observation period.

In 20 pregnant women of 32-42 weeks' gestation, the 4 components of fetal motor activity (head, trunk, arms, legs) were each computed, using two real time scanners, and correlated with the maternal subjective assessment of fetal activity. It was established that fetal trunk movements (FTM) correlated strongly with total fetal motor activity, especially in those movements described as major. Thus FTM could be used to establish normal parameters of fetal motor activity in the 3rd trimester.

In 21 normal pregnancies 24-hour profiles were obtained, by recording for 1 hour in 3 , providing a total of 168 hours for analysis. The mean percentage incidence of FTM was $18 \%$, with a mean of 29 moves/30-min observation period. There was a welldefined circadian variation (day/night $=16 / 22 \%$ ) which was the reverse of that for fetal respiratory movements (day $/$ night $=37 / 23 \%$ ). The variation in both parameters was wide but the total activity (breathing plus FTM) rarely fell below $10 \%$ in any 30-min study period.

Cross-sectional day-time data from 100 normal pregnancies between 28 weeks' gestation and term suggest that the mean number of moves/30-min period was inversely related to gestational age, although the percentage incidence of FTM (mean \pm $\mathrm{SD}=18 \pm 10 \%$ ) was constant throughout the 3rd trimester.

FTM was also studied in 20 pregnancies complicated by intrauterine growth retardation and 25 pregnancies complicated by maternal diabetes mellitus. Preliminary data from the latter group suggest that maternal hypoglycaemia may stimulate fetal motor activity.

\title{
Correspondence
}

\section{Epididymo-orchitis in Kawasaki disease}

\section{Sir,}

We were interested to read the report by Morgan and Lynch of a case of Kawasaki disease (Archives, 1978, 53, 916). We wish to report a hitherto unrecorded finding occurring during the course of this condition.

A 6-year-old boy was admitted to hospital because of a possible abscess in the left posterior cervical area. He had a pyrexia of $38 \cdot 5^{\circ} \mathrm{C}$. A confluent erythematous rash was present on his trunk and limbs, being more pronounced on the palms and soles. The left testis was slightly tender and enlarged. He had a strawberry tongue, and the veins of the bulbar conjunctiva were noted to be very prominent. The temperature returned to normal after 5 days and he then developed pain and swelling of the right wrist. At the same time there was a striking desquamation of the skin on the palmar aspect of his finger tips. The nail bed was not affected. The testicular tenderness which had been noted on admission became more marked during the next 3 days. Both testicle and epididymis became moderately enlarged. The skin of the scrotum was red and mildly oedematous. Symptomatic treatment only was offered, and after a week the testis and epididymis had returned to normal. At follow-up 6 weeks later no physical abnormalities were present.
This case fulfils the main criteria for diagnosis of Kawasaki disease suggested by Tanaka et al. (1976). There have been some less common associated findings in this condition. One of these is sterile leucocyturia, which was present in our case. It is almost certain that this is due to urethritis, as suprapubic aspiration carried out in the presence of pyuria in a number of these cases has shown normal bladder urine (Melish et al., 1976). However, testicular involvement has not thus far been described. It is likely that the inflammation in our case started in the urethra and progressed via the vas to involve the epididymis and testis.

\section{Reference}

Melish, M. E., Hicks, R. M., and Larson, E. J. (1976). Mucocutaneous lymph node syndrome in the United States. American Journal of Diseases of Children, 130, 599-607.

Tanaka, N., Seikimoto, K., and Naoe, S. (1976). Kawasaki disease. Relationship with infantile periarteritis nodosa. Archives of Pathology and Laboratory Medicine, 100, 81-86.

KeVin D. ConNolly AND DOLORES TIMMONS

Paediatric Unit,

Portiuncula Hospital, Ballinasloe,

Co. Galway, Eiré 